\title{
First overtone pulsators among Cepheids
}

\author{
L. Mantegazza ${ }^{1}$, E. Poretti ${ }^{2}$ \\ ${ }^{1}$ Università degli Studi, Pavia, Italy \\ ${ }^{2}$ Osservatorio Astronomico di Brera, Merate, Italy
}

The Fourier decomposition has been successfully applied to several classes of pul sating variables. Antonello and Poretti (1986) and Antonello et al. (1990a) applier it to the Cepheids with $P<8 \mathrm{~d}$. The latter authors redefined the $s$-Cepheids a Population I Cepheids that do not follow the Hertzsprung progression, but have progression of their own. The same authors proposed a new denomination (Antonell et al., 1990b): C-a stars to indicate the Classical Cepheids and C-b stars to indicat the redefined $s$-Cepheids.

The new photometric data obtained at La Silla and Merate Observatories (Man tegazza and Poretti, 1992) increase the evidence of a separation of Cepheids into tw well defined subclasses on the basis of the Fourier parameters of their light curves.

In the $\phi_{21}-P$ plane, the $s-$ and Classical Cepheids are characterized by tw sequences well separated for $\mathrm{P}<5.5 \mathrm{~d}$. In the period range $3 \mathrm{~d}<P<5.5 \mathrm{~d}$, tw different progressions are also present in the $\phi_{31}-P$ plane while a discriminatin value $R_{21}=0.20$ can be seen in the $R_{21}-P$ plane. In the $R_{31}-P$ plane th separation is well defined only for $P<4.5 \mathrm{~d}$, with a discriminating value $R_{31}=0.0 \varepsilon$

Besides the identification of new first overtone pulsators located on the uppe $s$-Cepheid sequence, we can point out that in the $\phi_{21}-P$ plane the increasing dis persion around $3 \mathrm{~d}$ strengthens the hypothesis that a resonance with a higher overton abruptly stops the regular u.pper progression; consequently the Cepheids located o the lower sequence should also be first overtone pulsators. The common nature of a these stars finds another observational support in the relationships noticeable in th $R_{21}-P, \phi_{31}-P, R_{31}-P$ planes, now better established on the basis of the ner available light curves.

\section{References:}

Antonello, E. and Poretti, E., 1986, Astron. Astrophys. 169, 149.

Antonello, E., Poretti, E., Reduzzi, L., 1990a, Astron. Astrophys. 236, 138.

Antonello, E., Poretti, E., Reduzzi, L., 1990b, in: Confrontation between Stellar Pulsatio and Evolution, Astr. Soc. of Pacific Conf. Series 11, p 209.

Mantegazza, L., Poretti, E., 1992, Astron. Astrophys., in press. 